\title{
The Importance of Antibodies and other Molecules as Biomarkers in Rheumatoid Arthritis
}

\author{
Maria Benito* \\ Clinical Medicine, IPN Communications, Dublin, Ireland \\ *Corresponding Author: Maria Benito, Clinical Medicine, IPN Communications, Dublin, Ireland.
}

Received: June 11, 2019; Published: July 30, 2019

DOI: $10.31080 /$ ASMS.2019.03.0367

\begin{abstract}
Rheumatoid Arthritis (RA) is a chronic inflammatory autoimmune disease characterized by synovitis, infiltrated inflammatory cells, cytokine production, and joint destruction. Some typicalbiomarkers are normally used to detect RA. However, these biomarkers are not exclusive of RA, as they might increase with age and gender, in tumours or infections. Therefore, it would be of diagnostic interest to find specific markers for RA.
\end{abstract}

Keywords: Rheumatoid Arthritis; Biomarkers; Erythrocyte Sedimentation Rate; C-Reactive Protein; Autoantibodies

\section{Abbreviations}

ACP: Anti-Citrullinated Peptide; ANA: Antinuclear Antibodies; antidsDNA: Anti-Double-Stranded DNA; CRP: C-Reactive Protein; ESR: Erythrocyte Sedimentation Rate; IgG: Immunoglobulin G; miRNAs: microRNAs; RA: Rheumatoid Arthritis; RF: Rheumatoid Factor; TNFi: Tumour Necrosis Factor Inhibitor; TNF- $\alpha$ : Tumour Necrosis Factor-Alpha.

\section{Introduction}

In many inflammatory disorders, it is well know the profile of cytokines and chemokines locally present in the affected tissues and most times also in the peripheral blood, which holds a significant role in diagnostics or further confirmation of the disease. However, they are not very useful when it comes to a distinctive diagnosis. Clinical serological and proteomic biomarkers, on the other hand, are used to confirm preliminary diagnosis and follow the effect of treatment [1].

In the case of rheumatoid arthritis (RA), -an autoimmune disease whose main feature is inflammation-, the most used biomarkers for diagnosis are acute phase proteins such as erythrocyte sedimentation rate (ESR) [2-4], and C-reactive protein (CRP) [5], and autoantibodies such as rheumatoid factor (RF) [6], anti- nuclear antibodies (ANA) and anti-citrullinated peptide (ACP) -in which arginines are enzimatically transformed into citrullines; this structural change make possible for the IgG antibodies to target the citrullinated peptides [7]. Patients with AR are divided into two different groups -ACP positive or negative- years before the onset of the disease $[8,9]$ and with differential disease progression [10].

These biomarkers are not exclusive of RA, as they might increase in cases of malignant tumours or infections, and in some cases are even related to age and gender.

Autoantibodies to cellular and nuclear antigens, such as ANAs and anti-double-stranded DNA (anti-dsDNA) -which result from the dysregulation of the immune system and can be associated with autoimmune diseases-, deserve a particular mention. Studies in RA suggest an association between therapeutic responses and both ANA and anti-dsDNA autoantibody [11], although some studies display controversial results [12]. Thus, a recent research shows that tumour necrosis factor inhibitor (TNFi) therapy in RA patients can induce ANAs and anti-dsDNA autoantibodies, or can revert from positive to negative these antibodies after treatments with abatacept -a biological therapy that targets T-cell activation [13]-, while RA patients treated with infiximab or adalimumab -antibodies that target tumour necrosis factor-alpha (TNF- $\alpha$ )- develop 
higher ANA and anti-dsDNA autoantibodies [14], highlighting the importance of T cells TNF- $\alpha$ in this process.

The use of autoantibodies - which should possess high sensitivity and specificity- in RA and other autoimmune disorders, not only allows for an early detection, but helps in the management of the disease and the right treatment. Things get complicated however, when these antibodies are present not only in affected patients but in healthy people too, which can lead to false positives and consequent unnecessary or incorrect treatments.

Most recently, microRNAs (miRNAs) -small non-coding RNAs involved in the regulation of gene expression at the posttranscriptional level- have also been investigated in RA as possible biomarkers. In a new study, quantitative expression of serum miRNA146a, miRNA-499 as well as their genotyping rs2910164 (C/G) and rs3746444 (T/C), respectively, were performed in RA patients with active and inactive RA using real-time PCR. Results showed that serum miRNA-146a and were significantly over expressed in RA patients and associated with RA protection and susceptibility respectively. Furthermore, miRNA-146a was negatively correlated with ANA [15]. Although this expression did not correlate with disease activity, results render miRNA-146a and miRNA-499 as diagnostic markers for RA.

\section{Conclusion}

Despite the pros and cons of the current antibodies and other type of biomarkers used, the diagnosis of RA is still based in symptoms and physical examination, but the use of biomarkers is a remarkable tool that facilitates and improves the management of RA.

\section{Acknowledgement}

\section{N/A}

\section{Conflict of Interest}

No financial interest or conflict of interest exists.

\section{Bibliography}

1. Aletaha D., et al. "Rheumatoid arthritis classification criteria: an American College of Rheumatology/ European League Against Rheumatism collaborative initiative". Arthritis and Rheumatology 62 (2010): 2569-2581.

2. Colglazier CL and Sutej PG. "Laboratory testing in the rheumatic diseases: a practical review". Southern Medical Journal 98 (2005): 185-191.
3. Neto R., et al. "The use of inflammatory laboratory tests in rheumatology". Revista Brasileira de Reumatologia 49 (2009): 413-430.

4. Sox HC and Liang MH. "The erythrocyte sedimentation rate. Guidelines for rational use". Annals of Internal Medicine 104 (1986): 515-523.

5. Gabay C and Kushner I. "Acute-phase proteins and other systemic responses to inflammation". The New England Journal of Medicine 340 (1999): 448-454.

6. Emery P., et al. "Evidence-based review of biologic markers as indicators of disease progression and remission in rheumatoid arthritis". Rheumatology International 27 (2007): 793-806.

7. Girbal-Neuhauser E., et al. "The epitopes targeted by the rheumatoid arthritis-associated antifilaggrin autoantibodies are posttranslationally generated on various sites of (pro)filaggrin by deimination of arginine residues". Journal of Immunology 162 (1999): 585-594.

8. Nielen MM., et al. "Specific autoantibodies precede the symptoms of rheumatoid arthritis: a study of serial measurements in blood donors". Arthritis and Rheumatology 50 (2004): 380386.

9. van der Helm-van Mil AH and Huizinga TW. "Advances in the genetics of rheumatoid arthritis point to subclassification into distinct disease subsets". Arthritis Research and Therapy 10 (2008): 205.

10. Bos WH., et al. "Arthritis development in patients with arthralgia is strongly associated with anti-citrullinated protein antibody status: a prospective cohort study". Annals of the Rheumatic Diseases 69 (2010): 490-494.

11. Takase K., et al. "What is the utility of routine ANA testing in predicting development of biological DMARD-induced lupus and vasculitis in patients with rheumatoid arthritis? Data from a single-centre cohort". Annals of the Rheumatic Diseases 73 (2014): 1695-1699.

12. Bacquet-Deschryver H., et al. "Impact of three anti-TNF alpha biologics on existing and emergent autoimmunity in rheumatoid arthritis and spondylarthropathy patients". Journal of Clinical Immunology 28 (2008): 445-55. 
13. Buch MH., et al. "Can switching to abatacept therapy in patients with rheumatoid arthritis on background methotrexate reverse TNF-inhibitor-induced antinuclear autoantibody/ double-stranded DNA autoantibody conversion? An analysis of the AMPLE and ATTEST trials". Clinical and Experimental Rheumatology (2018).

14. Charles PJ., et al. "Assessment of antibodies to double-stranded DNA induced in rheumatoid arthritis patients following treatment with infiximab, a monoclonal antibody to tumor necrosis factor alpha: findings in open label and randomized placebo-controlled trials". Arthritis and Rheumatology 43 (2000): 2383-90.

15. Ayeldeen G., et al. "Possible use of miRNAs-146a and -499 expression and their polymorphisms as diagnostic markers for rheumatoid arthritis". Molecular and Cellular Biochemistry (2018).

\section{Volume 3 Issue 8 August 2019}

(C) All rights are reserved by Maria Benito. 